\title{
Superconductivity from collective excitations in magic-angle twisted bilayer graphene
}

\author{
Girish Sharma, ${ }^{1,2,3}$ Maxim Trushin, ${ }^{1}$ Oleg P. Sushkov, ${ }^{4}$ Giovanni Vignale,${ }^{1,5,6}$ and Shaffique Adam $\odot^{1,2,5}$ \\ ${ }^{1}$ Centre for Advanced 2D Materials, National University of Singapore, 6 Science Drive 2, 117546 Singapore \\ ${ }^{2}$ Department of Physics, National University of Singapore, 2 Science Drive 3, 117551 Singapore \\ ${ }^{3}$ School of Basic Sciences, Indian Institute of Technology Mandi, Mandi 175005, India \\ ${ }^{4}$ School of Physics, The University of New South Wales, Sydney 2052, Australia \\ ${ }^{5}$ Yale-NUS College, 16 College Avenue West, 138527 Singapore \\ ${ }^{6}$ Department of Physics and Astronomy, University of Missouri, Columbia, Missouri 65211, USA
}

(Received 19 September 2019; revised manuscript received 27 February 2020; accepted 27 April 2020; published 13 May 2020)

\begin{abstract}
A purely electronic mechanism is proposed for the unconventional superconductivity recently observed in twisted bilayer graphene (tBG) close to the magic angle. Using the Migdal-Eliashberg framework on a one-parameter effective lattice model for $\mathrm{tBG}$ we show that a superconducting state can be achieved by means of collective electronic modes in tBG. We posit robust features of the theory, including an asymmetrical superconducting dome and the magnitude of the critical temperature that are in agreement with experiments.
\end{abstract}

DOI: 10.1103/PhysRevResearch.2.022040

Introduction. The remarkable experimental observations of superconducting and insulating phases in twisted bilayer graphene (tBG) [1-3] close to the magic angle usher in a new paradigm in attempts to study strongly correlated phases of matter by band-structure engineering. On stacking two graphene monolayers at a small relative twist angle $\theta$, a large-wavelength moiré superlattice potential emerges. Further, band calculations reveal [4] that there is substantial renormalization of the Fermi velocity giving rise to flat bands around half filling once $\theta$ is sufficiently small. At some very specific "magic angles" $\left(\theta_{M} \sim 1.1^{\circ}\right)$, the Fermi velocity is predicted to vanish, facilitating strong electronic correlations. It is therefore plausible that the superconducting instability observed in twisted bilayer graphene is a consequence of these correlations.

Given the rich underlying physics at play, a lot of theoretical effort has already been devoted towards understanding the Mott-like physics of strongly correlated electrons in tBG [5-17], and attributing the observed superconducting state to weak electron-phonon coupling [18-22]. However, it is not obvious that the latter is a conventional Bardeen-CooperSchriefer (BCS) state even though the phonon-mediated superconducting pairing is amplified in $\mathrm{tBG}$ due to the enhanced density of states at the Fermi surface (see Fig. 1). Neither is there a universal consensus or understanding that Mottlike physics is in action, further supported by the fact that recent experimental work has observed superconductivity independent of correlated insulating states [23-25]. In contrast, Coulomb interactions and associated plasmonic effects are

Published by the American Physical Society under the terms of the Creative Commons Attribution 4.0 International license. Further distribution of this work must maintain attribution to the author(s) and the published article's title, journal citation, and DOI. known to be very strong in graphene [26,27], and theoretically [28], may lead to a superconducting state. In this Rapid Communication we explore the possibility of unconventional superconductivity in $\mathrm{tBG}$ mediated by the purely collective electronic modes.

We first emphasize that the electronic mechanism is quite different from the standard BCS interaction, as no phonon modes are necessary. The collective oscillations can generate an effective dynamic attractive interaction, thus Cooper-pairing two electrons. Starting from a one-parameter effective lattice model for tBG [29] we first calculate the dynamical polarization function $\Pi(\mathbf{q}, i \omega)$ and thereby the dynamically screened Coulomb interaction $V(\mathbf{q}, i \omega)$. Following Grabowski and Sham [28], we average the interaction kernel to a dimensionless momentum-independent interaction parameter, $\lambda_{n m}$, and obtain the gap equation as follows:

$$
\Delta\left(i \omega_{n}\right)=-\frac{2 T_{C}}{E_{F}} \sum_{m} \frac{\arctan \left[E_{F} /\left(Z_{m} \omega_{m}\right)\right]}{\left(Z_{m} \omega_{m}\right) / E_{F}} \lambda_{n m} \Delta\left(i \omega_{m}\right),
$$

where $\omega_{n}=(2 n+1) \pi T_{C}$ is the Matsubara frequency, and $Z_{m}$ is a renormalizing function calculated below. Solving Eq. (1) we find the superconducting critical temperature $T_{C}$ for various twist angles $\theta$ and carrier concentrations linked to the Fermi energy $E_{F}$ via density of states. The prominent predictions of our theory are as follows: (i) the plasmon-assisted Cooper pairing is much stronger than the conventional phonon-related one in tBG, (ii) the superconducting state does not occur at low electron concentrations but is prominent at electron densities around $n=10^{12} \mathrm{~cm}^{-2}$, (iii) the obtained critical temperature is of $O(10 \mathrm{~K})$, similar to that observed in experiments [1-3] and larger than those calculated by the phonon contribution [18], and (iv) our theory is not limited by particular plasmon modes as our model generically captures the effect of plasmons (whenever well defined), but also the other density-fluctuation excitations. 

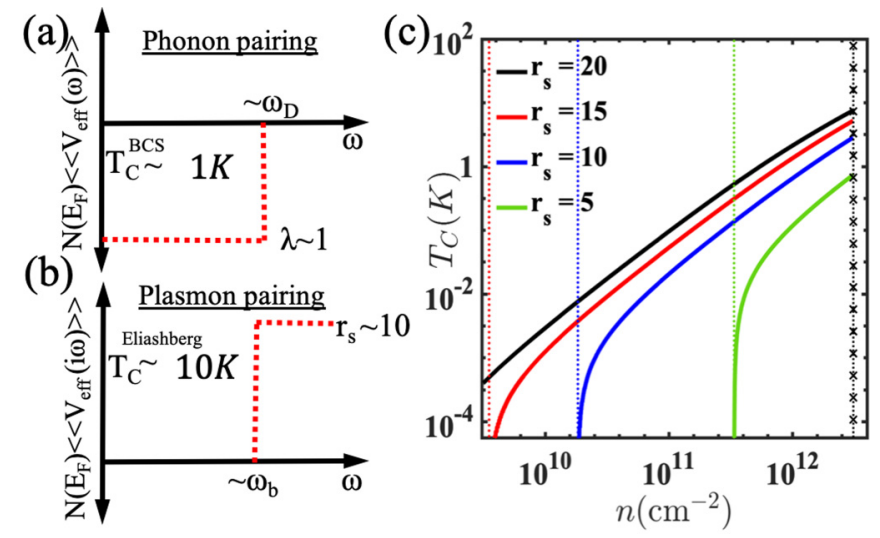

FIG. 1. Cartoon illustration of a mechanism for superconductivity: (a) within BCS theory, moiré phonons [18] have an attractive pairing below the Debye frequency $\omega_{D}$; (b) although plasmonic mediated interaction is repulsive, the frequency dependence gives rise to pairing within Eliashberg theory; (c) analytic single-mode approximation [Eq. (2)] for the Dirac model with renormalized $v_{F} \sim 1.5 \times 10^{4} \mathrm{~ms}^{-1}, \omega_{b} \sim 15 \mathrm{meV}$ gives a $T_{C}$ for a finite density window between the dotted and the crossed lines. The dotted lines indicate the threshold density and the crossed black line indicates the cutoff imposed by the finite bandwidth. The enhanced density window supporting superconductivity with increasing $r_{s}$ agrees well with the full numerical results.

Phonons or plasmons? The striking scenario which develops at small twist angles is that the relevant electronic energy scales are shrunk to the order of a few meV (similar to the acoustic phonon energy scale), while the density of states is amplified. This raises the intriguing possibility of observing collective electronic modes in the same energy window. For example, it was recently [29] pointed out that plasmon modes in $\mathrm{tBG}$ can remain intrinsically undamped protected by the band gap between the flat bands and higher bands. It is also worth noting the possibility to have hybrid acoustic phonon and plasmon modes still uncommon in condensed matter systems.

Let us compare the phonon- and plasmon-mediated superconductivity mechanisms in tBG with $\theta$ close to $\theta_{M}$. Here, we assume a simplest two-dimensional massless Dirac fermion model with the renormalized Fermi velocity $v_{F}$. The Fermi surface is then just a circle of radius $k_{F}$, and the averaged electron-phonon coupling constant is $\lambda=$ $\zeta^{2} \pi k_{F} /\left(\hbar v_{F} \rho c_{\mathrm{ph}}^{2}\right) \sim O(1)$, where $\zeta, \rho$, and $c_{\mathrm{ph}}$ are deformation potential, mass density, and the sound velocity, respectively. To the first approximation we can assume the attractive pairing potential to be finite only below the Debye frequency $\omega_{D}$ [see Fig. 1(a)]. The McMillan formula then suggests $T_{C} \sim \omega_{D} \exp (-1 / \lambda) \sim O(1 \mathrm{~K})$, as also shown recently [18]. In contrast, the plasmon-mediated mechanism suggests that the dynamic Coulomb interaction $V(\mathbf{q}, i \omega)$ is responsible for the superconducting pairing. For such pairing the dynamical nature of the coupling is crucial because the Coulomb interaction is screened by the dielectric function $\epsilon(q, i \omega)=$ $1+e^{2} E_{F} q /\left(2 \kappa \omega^{2}\right)[30,31]$ providing stronger interactions in the high-frequency limit. Here, the renormalized dielectric constant $\kappa \sim 12$ accounts for effects of interband polarization in tBG [29]. The interaction strength can also be tuned by dielectric engineering [32]. The corresponding dimensionless coupling $\lambda_{n m}$ reduces to the bare Coulomb interaction characterized by $r_{s}=e^{2} /\left(\kappa \hbar v_{F}\right)$ in the high-frequency limit, while remaining zero below a certain energy determined by $\omega_{b}[28,33]$. This behavior being quite opposite to the phononassisted pairing is schematically shown in Fig. 1(b).

Equation (1) is still too complicated, as it in fact represents an infinite number of coupled equations. We design a simple analytically tractable model by reducing the number of equations to three considering only the terms with $m=0, \pm M$, where $M \gg 1$. Neglecting the self-energy corrections for now (these will be included later on) and setting the diagonal elements $\lambda_{m m}=0$ we arrive at the following equation for $T_{C}$ [34]:

$$
r_{s} \frac{2 T_{C}}{\omega_{M}} \arctan \left(\frac{E_{F}}{\omega_{M}}\right)\left[\frac{4 r_{s}}{\pi} \arctan \left(\frac{E_{F}}{\pi T_{C}}\right)-1\right]=1 .
$$

We use the single-mode equation (2) with $M \sim \omega_{b} / E_{F}$ to estimate $T_{C}$ in Fig. 1(c). In the low- $T_{C}$ limit $\left(T_{C} \ll E_{F}\right)$ and strong coupling $\left(r_{s} \gg 1\right)$, we find an elegant expression

$$
\arctan \left(\frac{E_{F}^{2}}{2 \pi \omega_{b} T_{C}}\right)=\frac{\pi}{2} \frac{\omega_{b}}{r_{s}^{2} E_{F}},
$$

that can be seen as a plasmonic analog of the McMillan formula for $T_{C}$. Since the left-hand side of Eq. (3) cannot exceed $\pi / 2$, the solution for $T_{C}$ exists if and only if $\omega_{b} /\left(r_{s}^{2} E_{F}\right)<1$, i.e., the electron concentration and coupling strength values must be large enough. This is indeed the case in tBG. Estimating $\omega_{b} \sim 15 \mathrm{meV}, v_{F} \sim 1.5 \times 10^{4} \mathrm{~m} / \mathrm{s}$, we find $r_{s} \sim 12, E_{F}=\hbar v_{F} \sqrt{\pi n / 2} \sim 1.5 \mathrm{meV}$ (for electron density $n \sim 1.5 \times 10^{12} \mathrm{~cm}^{-2}$ ), and $M \sim 10$, resulting in a reasonable value $T_{C} \sim 2.6 \mathrm{~K}$ matching the observations [1]. In conventional graphene $r_{s} \sim 1, \omega_{b}>E_{F}$ [31], and solution of Eq. (3) never exists making the plasmon model tBG specific. This is consistent with the fact that superconductivity has been observed in tBG but not in monolayer graphene. The fact that using comparable approximations the plasmonic $T_{C}$ is estimated to be larger than the phononic one, suggests that plasmon-assisted pairing, which we investigate in detail below, is relevant for the observed experimental superconductivity.

Dynamical Coulomb interaction. To go beyond the singlemode model, we consider the following one-parameter effective nearest-neighbor tight-binding Hamiltonian defined on a hexagonal lattice, which mimics the low-energy Hamiltonian of twisted bilayer graphene [29]

$$
\begin{aligned}
H & =\sum_{\mathbf{k}} h_{\mathbf{k}} c_{\mathbf{k}, 2}^{\dagger} c_{\mathbf{k}, 1}+h_{\mathbf{k}}^{*} c_{\mathbf{k}, 1}^{\dagger} c_{\mathbf{k}, 2}, \\
h_{\mathbf{k}} & =t_{\mathrm{eff}} \sum_{\delta_{\mathbf{j}}} e^{i \mathbf{k} \cdot \delta_{\mathbf{j}}},
\end{aligned}
$$

where $c_{\mathbf{k}, \eta}$ and $c_{\mathbf{k}, \eta}^{\dagger}$ are the annihilation and creation operators for electrons with momentum $\mathbf{k}$ in the Brillouin zone on the sublattice $\eta$. The effective hopping matrix element $t_{\text {eff }}=W / 3$ corresponds to the bandwidth $(W)$ of the nearly flat bands in tBG close to the magic angle. The summation $\delta_{\mathbf{j}}$ is over the nearest neighbors $\delta_{\mathbf{j}}=[\cos (2 \pi j / 3), \sin (2 \pi j / 3)] \tilde{a} \sqrt{3}$, where 
$\tilde{a}=[a / 2 \sin (\theta / 2)]$ is the periodicity of the moiré superlattice. The constants $a=2.46 \AA$ is the graphene lattice constant, while $\theta$ is the twist angle. For our calculations we obtain the bandwidth $W$ from the tBG continuum model [4]. The advantage of the above tight-binding model is that it reproduces the symmetry of the actual tBG and has a natural ultraviolet smooth cutoff scale $W$. The divergent density of states at the van Hove singularity is also manifested in this model, which will be important for our analysis. The eigenvalues are $E_{\mathbf{k}}^{n}=n\left|h_{\mathbf{k}}\right|$ and the fourfold degenerate eigenstates are $\psi_{n, \mathbf{k}}^{\dagger}=$ $\left(2^{-1 / 2}\right)\left[e^{-i n \arg \left(h_{\mathbf{k}}\right)}, 1\right]$, where $n= \pm 1$ is the band index.

We are particularly interested in the dynamic polarization function $\Pi(\mathbf{q}, i \omega)$, which is given by

$$
\Pi(\mathbf{q}, i \omega)=4 \sum_{\mathbf{k}} \sum_{m, n} \frac{\left(f_{\mathbf{k}+\mathbf{q}}^{n}-f_{\mathbf{k}}^{m}\right) F_{\mathbf{k}, \mathbf{k}+\mathbf{q}}^{n m}}{E_{\mathbf{k}+\mathbf{q}}^{n}-E_{\mathbf{k}}^{m}-i \omega},
$$

where $n$ and $m$ are the band indices, $f_{\mathbf{k}}^{n}$ is the Fermi-Dirac distribution, and $F_{\mathbf{k}, \mathbf{k}+\mathbf{q}}^{n m}=\left|\psi_{n, \mathbf{k}+\mathbf{q}}^{\dagger} \psi_{m, \mathbf{k}}\right|^{2}$ is the graphene chirality factor. Since $h_{\mathbf{k}}$ has a complicated momentum dependence, we evaluate the above function numerically expanding up to second order in q. As recently shown [29], this is actually sufficient to describe collective excitations such as plasmons over a wide range of energy scales $0<\omega<2 W$. The dynamical polarizability is used to evaluate the dielectric constant within the random phase approximation as $\epsilon(\mathbf{q}, i \omega)=1-$ $V_{\mathbf{q}} \Pi(\mathbf{q}, i \omega)$, where $V_{\mathbf{q}}=2 \pi e^{2} / \kappa q$ is the bare Coulomb interaction. Finally, the dynamically screened Coulomb interaction is given by $V(\mathbf{q}, i \omega)=V(\mathbf{q}) / \epsilon(\mathbf{q}, i \omega)$.

The gap equation. The Migdal-Eliashberg superconductivity theory suggests the following gap equation [35]:

$$
\begin{aligned}
\Delta\left(\mathbf{p}, i \omega_{n}\right)= & -T_{C} \sum_{\mathbf{k}, m}\left[V\left(\mathbf{p}-\mathbf{k}, i \omega_{n}-\omega_{m}\right) B\left(\mathbf{k}, i \omega_{m}\right)\right. \\
& \left.\times \Delta\left(\mathbf{k}, i \omega_{m}\right)\right],
\end{aligned}
$$

where the product of the Green's functions $B\left(\mathbf{k}, i \omega_{m}\right)$ is given by

$$
\begin{aligned}
& B\left(\mathbf{k}, i \omega_{m}\right)=G\left(\mathbf{k}, i \omega_{m}\right) G\left(-\mathbf{k},-i \omega_{m}\right), \\
& G\left(\mathbf{k}, i \omega_{m}\right)=\frac{1}{i \omega_{m}-\epsilon_{k}-\Sigma\left(\mathbf{k}, i \omega_{m}\right)} .
\end{aligned}
$$

Here, $\Sigma\left(\mathbf{k}, i \omega_{m}\right)$ represents the normal-state self-energy, which is given by

$$
\Sigma\left(\mathbf{p}, i \omega_{n}\right)=-T_{C} \sum_{m \mathbf{k}} \frac{V\left(\mathbf{p}-\mathbf{k}, i \omega_{n}-i \omega_{m}\right)}{i \omega_{m}-\epsilon_{\mathbf{k}}-\Sigma\left(\mathbf{k}, i \omega_{m}\right)} .
$$

In the above expressions the energy dispersion $\epsilon_{\mathbf{k}}$ is measured from the Fermi energy. Note that we will now restrict our attention to the conduction band intersecting the Fermi energy relevant for an electron-doped system. We point out that one may include the phonon contribution in this framework by adding the phononic propagator $D(\mathbf{q}, \omega)$ into the gap equations. Since we are interested in evaluating the pure electronic contribution, we do not attempt this calculation here and reserve it for future studies. In the Supplemental Material [34] we do present a Migdal-Eliashberg calculation for a pure phonon mechanism.

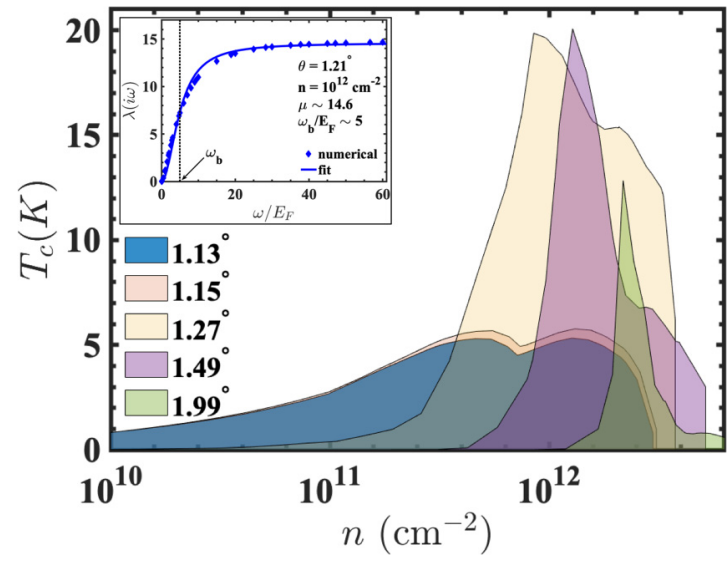

FIG. 2. Critical temperature $T_{C}$ as a function of electron density in $\mathrm{tBG}$ (close to the magic angle). The presence of an asymmetrical superconducting dome around $n=10^{12} \mathrm{~cm}^{-2}$ and $T_{C}=O(10 \mathrm{~K})$ are the main predictions of this theory. The inset shows a comparison of numerically obtained $\lambda(i \omega)$ and the fit using Eq. (10). The evaluated parameters $\mu$ and $\omega_{b}$ are shown, and the position of $\omega_{b}$ is indicated by the dotted line.

It has been shown [28] that superconductivity in a Coulomb system with no attractive interactions is essentially determined by the frequency dependence of the screened Coulomb interaction. Therefore, we can work with an effective Coulomb interaction, which is averaged over the momenta up to $\mathbf{k}_{c}=2 \mathbf{k}_{F}$ [28], thus retaining only the crucial frequency dependence. The momentum-averaged interaction is given by

$$
\left\langle\left\langle V\left(i \omega_{l}\right)\right\rangle\right\rangle=\frac{\sum_{\mathbf{k}, \mathbf{p}} \Theta\left(\mathbf{k}_{c}-\mathbf{k}\right) \Theta\left(\mathbf{k}_{c}-\mathbf{p}\right) V\left(\mathbf{p}-\mathbf{k}, i \omega_{l}\right)}{\sum_{\mathbf{k}, \mathbf{p}} \Theta\left(\mathbf{k}_{c}-\mathbf{k}\right) \Theta\left(\mathbf{k}_{c}-\mathbf{p}\right)} .
$$

The dimensionless coupling is given by $\lambda(i \omega)=$ $\langle\langle V(i \omega)\rangle\rangle N\left(E_{F}\right)$, where $N\left(E_{F}\right)$ is the density of states. The limiting cases of the coupling are given by $\lim _{\omega=0}\langle\langle V(i \omega)\rangle\rangle N\left(E_{F}\right) \rightarrow 0$ and $\lim _{\omega \rightarrow \infty}\langle\langle V(i \omega)\rangle\rangle N\left(E_{F}\right)=$ $\mu$, where we define $\mu$ to be the high-frequency limit of the coupling. To proceed further, the dimensionless coupling is then mapped onto the following explicit expression:

$$
\begin{aligned}
\lambda_{n m} & =\left\langle\left\langle V\left(i \omega_{n}-i \omega_{m}\right)\right\rangle\right\rangle N\left(E_{F}\right) \\
& =\mu\left(1-\frac{\omega_{b}^{2}}{\left(\omega_{n}-\omega_{m}\right)^{2}+\omega_{b}^{2}}\right),
\end{aligned}
$$

where $\omega_{b}$ is a boson frequency that sets the scale of transition from the low-frequency to the high-frequency limit. The mapping of the kernel onto the above Lorentzian form allows for an analytical treatment that is indeed found to closely resemble the actual numerical solution for $\lambda_{n m}$ (see inset of Fig. 2). The parameters $\mu$ and $\omega_{b}$ are then extracted by fitting the actual coupling $\lambda(i \omega)$ to Eq. (10). The propagating boson here (with frequency $\omega_{b}$ ) is a collective density-fluctuation excitation such as electron-hole excitation or a plasmon. The inset in Fig. 3 plots $\omega_{b}$ as a function of $\theta$ and compares 


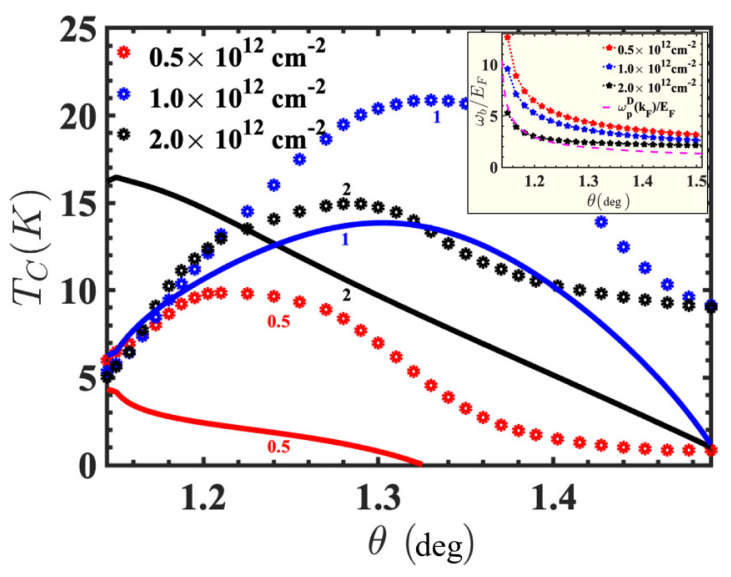

FIG. 3. Critical temperature $T_{C}$ as a function of twist angle in tBG for three different carrier densities. The dotted lines show the $T_{C}$ obtained from the full numerical solution of Eq. (1). The analytical solution from Eq. (2) using the parameters $\omega_{b}, E_{F}$, and $\mu$ (or $r_{s}$ ) from the numerical solution is shown by corresponding solid lines. The inset shows $\omega_{b}$ as a function of $\theta$ determined by fitting the numerical calculation of $\lambda(i \omega)$. The dashed line shows, for comparison, $\omega_{p}^{D}\left(k_{F}\right)$, the plasmon frequency within a Dirac model which has a qualitatively similar enhancement at low twist angles.

it to the plasmon dispersion at $k_{F}$ within the Dirac model. For the momentum-averaged interaction, Eq. (6) becomes momentum independent and is given by Eq. (1) with $Z_{n}=1+$ $\mu\left(\omega_{b} / \omega_{n}\right) \arctan \left\{\omega_{n} E_{F} /\left[\left(\omega_{n}^{2}+\omega_{b}\left(E_{F}+\omega_{b}\right)\right]\right\}\right.$ accounting for the self-energy corrections.

The gap equation is now of the form of an eigenvalue equation $\bar{\Delta}=\hat{C} \bar{\Delta}$. At $T_{C}$ the largest eigenvalue of $\hat{C}$ is exactly 1 . Equation (1) must be solved numerically to obtain a reliable value for critical temperature. The rate of convergence of the numerical solution depends on the ratio $T_{C} / E_{F}$. If $T_{C} \ll E_{F}$, the dimensions of the matrix involved can become prohibitively large to allow a numerical solution. However, in this regime the pseudopotential method [28], which assumes $T_{C} \ll E_{F}$ as a premise, gives us a good estimate of the superconducting critical temperature. For our calculations we use a combination of numerical solution and pseudopotential method depending on the ratio $T_{C} / E_{F}$. We also briefly comment on the nature of the superconducting gap function $\Delta(i \omega)$. Since we have purely repulsive interactions (unlike the case of phonon-mediated BCS coupling), the gap function changes sign as a function of the Matsubara frequency. This is necessary for the gap equations to have a nontrivial solution. In the Supplemental Material [34] we solve the momentumdependent Elaishberg equations and show that the pairing symmetry is mainly $s$ wave, consistent with the premise of our calculation.

Superconductivity. We will now discuss salient predictions of our theory. In Fig. 2 we plot the critical temperature $T_{C}$ as a function of electron density in $\mathrm{tBG}$ close to the magic angle. The presence of an asymmetrical superconducting dome around $n=10^{12} \mathrm{~cm}^{-2}$ and the calculated $T_{C}=O(10 \mathrm{~K})$ are the main predictions of our theory. The obtained $T_{C}$ closely resembles the measured order of magnitude in experiments [1-3], and is also larger than the calculated $T_{C}$ from the phonon contribution [18]. As the carrier density is increased the first maximum of the superconducting dome occurs when the Fermi energy intersects the $\mathbf{M}$ of the Brillouin zone point, where the van Hove singularity occurs. As the density is increased further, the dome is found to be asymmetric around this point, which can be understood from the fact that the density of states is not symmetric around the $\mathbf{M}$ point. This asymmetry combined with the effect of strong electronic interactions results in a second neighboring maximum in the dome [34], which is more prominent at lower twist angles. When $\theta$ is increased further the width of the dome shrinks and the second maximum becomes less prominent since the electronic interactions become comparatively weaker. We observe superconductivity even close to $\theta \sim 2^{\circ}$, but the corresponding density window is quite narrow. In the monolayer limit the superconducting dome is practically nonexistent within our model. The appearance of these domes is a special feature which arises from the chosen realistic lattice model as opposed to the simple Dirac approximation for tBG. By examining the dependence on the dielectric constant, we notice that the magnitude of $T_{C}$ is set primarily by $r_{s}$ (or $\mu$ ), while the overall shape of the domes is set by the density of states of the noninteracting bands. Also note that we specifically focus on superconductivity. Other competing states (such as density waves, magnetism, etc.) may obviously affect the superconducting dome, when taken into consideration. In Fig. 3 we plot the obtained critical temperature $T_{C}$ as a function of twist angle for different carrier densities. The dependence is observed to be nonmonotonic, and for large angles $T_{C}$ is eventually suppressed, as expected. Figure 3 also compares the numerical solution to the $T_{C}$ obtained from the singlemode model in Eq. (2). In contrast to our findings, $T_{C}$ from the phonon mechanism [18] is noted to increase with $\theta$, and oscillate with respect to the filling fraction. This is because the electron-phonon coupling is shown to rapidly increase as a function of density.

Concluding remarks. We first point out that the vertex corrections are neglected in the Migdal-Eliashberg formalism, which may have a quantitative impact on the calculated $T_{C}$. For twist angles close to the magic angle, we find that $\omega_{b}$ can be several times larger than the Fermi energy $E_{F}$. However in this regime, the vertex corrections can be ignored, because they turn out to be insignificant for processes much larger than $E_{F}$ as pointed out earlier in the literature [36]. Therefore the calculated $O\left(T_{C}\right)$ is expected to be robust especially close to the interesting regime of the magic angle. Vertex corrections may become more important for large twist angles causing suppression of $T_{C}$, but evaluating them is beyond the scope of the current Rapid Communication.

To conclude, this work specifically points out an important mechanism which is likely to be at play in superconducting $\mathrm{tBG}$ close to the magic angle. Collective excitations of strongly coupled electrons can mediate pairing, which may be dubbed as "plasmonic superconductivity," although the requirement of undamped plasmons is not strict in our formalism. We predict features of the theory, namely, the superconducting dome and the magnitude of $T_{C}$, which are in good agreement with recent experimental data [1,3]. The nature of the gap function in momentum space is also inferred to be $s$ wave by solving the Eliashberg equations without any momentum averaging [34]. 
Acknowledgments. G.S. acknowledges useful discussions with D. Y. H. Ho, N. Raghuvanshi, H-K. Tang, I. Yudhistira, N. Chakraborty, M. M. E. AlEzzi, X. Gu, J. N. Leaw, and C. Setty. This work was supported by the Singapore Ministry of Education AcRF Tier 2 Grants No. MOE2017-T2-2-140 and No. MOE2017-T2-1-130. M.T. acknowledges the Director's Senior Research Fellowship from the Centre for Advanced 2D Materials at the National University of Singapore (Singapore
NRF Grants No. R-723-000-001-281 and No. R-607-000352-112). O.P.S. and S.A. are supported by the Australian Research Council Centre of Excellence in Future Low-Energy Electronics Technologies (CE170100039).

G.S. performed the calculations with input from M.T., G.V., and S.A. M.T. devised the single-mode model. The project was conceived by G.S., O.P.S., G.V., and S.A.
[1] Y. Cao, V. Fatemi, S. Fang, K. Watanabe, T. Taniguchi, E. Kaxiras, and P. Jarillo-Herrero, Nature (London) 556, 43 (2018).

[2] Y. Cao, V. Fatemi, A. Demir, S. Fang, S. L. Tomarken, J. Y. Luo, J. D. Sanchez-Yamagishi, K. Watanabe, T. Taniguchi, E. Kaxiras et al., Nature (London) 556, 80 (2018).

[3] M. Yankowitz, S. Chen, H. Polshyn, Y. Zhang, K. Watanabe, T. Taniguchi, D. Graf, A. F. Young, and C. R. Dean, Science 363, 1059 (2019).

[4] R. Bistritzer and A. H. MacDonald, Proc. Nat. Acad. Sci. USA 108, 12233 (2011).

[5] C. Xu and L. Balents, Phys. Rev. Lett. 121, 087001 (2018).

[6] B. Roy and V. Juričić, Phys. Rev. B 99, 121407(R) (2019).

[7] H. C. Po, L. Zou, A. Vishwanath, and T. Senthil, Phys. Rev. X 8, 031089 (2018).

[8] M. Koshino, N. F. Q. Yuan, T. Koretsune, M. Ochi, K. Kuroki, and L. Fu, Phys. Rev. X 8, 031087 (2018).

[9] J. Kang and O. Vafek, Phys. Rev. X 8, 031088 (2018).

[10] B. Padhi, C. Setty, and P. W. Phillips, Nano Lett. 18, 6175 (2018).

[11] H. Guo, X. Zhu, S. Feng, and R. T. Scalettar, Phys. Rev. B 97, 235453 (2018).

[12] C.-C. Liu, L.-D. Zhang, W.-Q. Chen, and F. Yang, Phys. Rev. Lett. 121, 217001 (2018).

[13] H. Isobe, N. F. Q. Yuan, and L. Fu, Phys. Rev. X 8, 041041 (2018).

[14] Y.-Z. You and A. Vishwanath, npj Quantum Mater. 4, 16 (2019).

[15] J. González and T. Stauber, Phys. Rev. Lett. 122, 026801 (2019).

[16] M. Xie and A. H. MacDonald, Phys. Rev. Lett. 124, 097601 (2020).

[17] E. Laksono, J. N. Leaw, A. Reaves, M. Singh, X. Wang, S. Adam, and X. Gu, Solid State Commun. 282, 38 (2018).

[18] B. Lian, Z. Wang, and B. A. Bernevig, Phys. Rev. Lett. 122, 257002 (2019).
[19] Y. W. Choi and H. J. Choi, Phys. Rev. B 98, 241412(R) (2018).

[20] F. Wu, A. H. MacDonald, and I. Martin, Phys. Rev. Lett. 121, 257001 (2018)

[21] I. Yudhistira, N. Chakraborty, G. Sharma, D. Y. H. Ho, E. Laksono, O. P. Sushkov, G. Vignale, and S. Adam, Phys. Rev. B 99, 140302(R) (2019).

[22] F. Wu, E. Hwang, and S. Das Sarma, Phys. Rev. B 99, 165112 (2019).

[23] H. S. Arora, R. Polski, Y. Zhang, A. Thomson, Y. Choi, H. Kim, Z. Lin, I. Z. Wilson, X. Xu, J.-H. Chu et al., arXiv:2002.03003.

[24] P. Stepanov, I. Das, X. Lu, A. Fahimniya, K. Watanabe, T. Taniguchi, F. H. Koppens, J. Lischner, L. Levitov, and D. K. Efetov, arXiv:1911.09198.

[25] Y. Saito, J. Ge, K. Watanabe, T. Taniguchi, and A. F. Young, arXiv:1911.13302.

[26] A. Grigorenko, M. Polini, and K. Novoselov, Nat. Photonics 6, 749 (2012).

[27] N. C. H. Hesp, I. Torre, D. Rodan-Legrain, P. Novelli, Y. Cao, S. Carr, S. Fang, P. Stepanov, D. Barcons-Ruiz, H. HerzigSheinfux et al., arXiv:1910.07893.

[28] M. Grabowski and L. J. Sham, Phys. Rev. B 29, 6132 (1984).

[29] C. Lewandowski and L. Levitov, Proc. Natl. Acad. Sci. U.S.A. 116, 20869 (2019)

[30] B. Wunsch, T. Stauber, F. Sols, and F. Guinea, New J. Phys. 8, 318 (2006)

[31] E. H. Hwang and S. Das Sarma, Phys. Rev. B 75, 205418 (2007).

[32] J. M. Pizarro, M. Rösner, R. Thomale, R. Valentí, and T. O. Wehling, Phys. Rev. B 100, 161102(R) (2019).

[33] G. S. Canright and G. Vignale, Phys. Rev. B 39, 2740 (1989).

[34] See Supplemental Material at http://link.aps.org/supplemental/ 10.1103/PhysRevResearch.2.022040 for calculation details.

[35] G. M. Eliashberg, Sov. Phys. JETP 11, 696 (1960).

[36] Y. Takada, J. Phys. Soc. Jpn. 61, 3849 (1992). 